\title{
KOALA: a wide-field, 1000 element integral-field unit for the Anglo-Australian Telescope
}

\author{
S.C. Ellis ${ }^{a}$, M. Ireland ${ }^{a, b}$, J.S. Lawrence ${ }^{a}$, J. Tims $^{a}$, N. Staszak ${ }^{a}$, J. Brzeski ${ }^{a}$, Q.A Parker ${ }^{a, b}$, R.

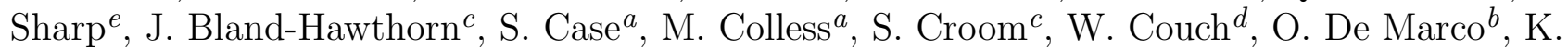 \\ Glazebrook $^{d}$, W. Saunders ${ }^{a}$, R. Webster ${ }^{f}$, D.B. Zucker ${ }^{b}$, \\ ${ }^{a}$ Australian Astronomical Observatory, P.O. Box 296, Epping, NSW 1710, Australia; \\ ${ }^{b}$ Department of Physics and Astronomy, Macquarie University, NSW 2109, Australia; \\ ${ }^{c}$ Sydney Institute for Astronomy, School of Physics, University of Sydney, NSW 2006, \\ Australia; \\ ${ }^{d}$ Centre for Astrophysics and Supercomputing, Swinburne University of Technology, PO Box \\ 218, Hawthorn, VIC 3122, Australia; \\ ${ }^{e}$ Research School of Astronomy and Astrophysics, Australian National University, Weston \\ Creek, ACT 2611, Australia; \\ ${ }^{f}$ School of Physics, University of Melbourne, Parkville, Victoria 3010, Australia
}

\begin{abstract}
KOALA, the Kilofibre Optimised Astronomical Lenslet Array, is a wide-field, high efficiency integral field unit being designed for use with the bench mounted AAOmega spectrograph on the AAT. KOALA will have 1000 fibres in a rectangular array with a selectable field of view of either 1390 or 430 sq. arcseconds with a spatial sampling of 1.25 " or 0.7 " respectively. To achieve this KOALA will use a telecentric double lenslet array with interchangeable fore-optics. The IFU will feed AAOmega via a $31 \mathrm{~m}$ fibre run. The efficiency of KOALA is expected to be $\approx 52 \%$ at $3700 \mathrm{~A}$ and $\approx 66 \%$ at $6563 \AA$ with a throughput of $>52 \%$ over the entire wavelength range.
\end{abstract}

Keywords: integral-field spectroscopy, optical spectrographs

\section{INTRODUCTION}

KOALA is a replacement for the current integral-field unit (IFU) on the Anglo-Australian Telescope (AAT), SPIRAL. SPIRAL ${ }^{1}$ samples the $\mathrm{f} / 8$ Cassegrain focal plane with a 512 element array of square microlenses in a $32 \times 16$ format, with a field of view of $22 \times 11$ arcseconds. SPIRAL was an upgrade to a prototype IFU, SPIRAL-A, ${ }^{2,3}$ and originally both incarnations used the same purpose built bench mounted spectrograph, until SPIRAL was decommissioned.

SPIRAL was recommissioned in 2006, this time feeding the AAOmega bench mounted spectrograph. ${ }^{4-6}$ Despite the somewhat ad hoc retro-fitting of the SPIRAL IFU to AAOmega, the instrument has been popular and successful. Highlights of the science done with SPIRAL include observations of local galaxies with a high level of turbulence caused by high star-formation rates, ${ }^{7}$ and the observation that star-burst galactic winds are shock ionised (whereas AGN galactic winds in the same sample are photoionised), indicating a delay between the onset of star-formation and the development of a wind. ${ }^{8}$ These examples reflect a growing demand for integral-field spectroscopy, which has proved itself to be a powerful technique for measuring the complex kinematic, structural and chemical properties of extended objects (e.g. SAURON; ${ }^{9}$ ATLAS $^{3 \mathrm{D} 10}$ ).

Nevertheless using SPIRAL with AAOmega has some significant limitations. In particular the current fibres have poor transmission below $4200 \AA$ making it impossible to measure either [OII] at $3727 \AA$ or the $\mathrm{Ca} \mathrm{H}+\mathrm{K}$

Further author information: (Send correspondence to S.C.E)

S.C.E.: E-mail: sellis@aao.gov.au, Telephone: +61 283724845

Ground-based and Airborne Instrumentation for Astronomy IV, edited by lan S. McLean, Suzanne K. Ramsay, Hideki Takami, Proc. of SPIE Vol. 8446, 84460V · (c) 2012 SPIE · CCC code: 0277-786X/12/\$18 · doi: 10.1117/12.925812 
Table 1. Properties of the KOALA instrument with the AAOmega spectrograph.

\begin{tabular}{ll}
\hline & \multicolumn{1}{c}{ KOALA } \\
\hline \hline Number of elements & 1000 \\
Format & $40 \times 25$ \\
Spatial resolution & 0.7 or $1.25 "$ \\
Field of view & $24 " \times 18 "=434$ sq. arcsec. or $43 " \times 32 "=1385$ sq. arcsec \\
Throughput $(3727 \AA)$ & 0.52 \\
Throughput $(6562 \AA)$ & 0.66 \\
\hline & \multicolumn{1}{c}{ AAOmega } \\
\hline Total wavelength coverage & $3700-9500 \AA$ \\
Spectral resolution & $1300-10000$ \\
Single shot wavelength coverage & $400-3200 \AA$
\end{tabular}

absorption lines and the $4000 \AA$ break; features which are crucial diagnostics for stellar population work. In addition the current fibre run is too short to allow the full range of movement of the telescope.

KOALA is a replacement IFU and fibre run, to feed AAOmega, that will resolve these problems. The overall efficiency will be increased by a factor of $\approx 1.3$. Furthermore KOALA will also have 1.8 or 5.7 times the field of view, depending on the spatial resolution, and twice number of elements of SPIRAL with a new IFU. KOALA is currently in the design phase, but is expected to be completed by June 2013. This paper describes the instrument and the expected performance.

\section{TECHNICAL DESCRIPTION}

KOALA is comprised of three sub components: the fore-optics which accept light from the telescope focal plane and feed it to the lenslet array, the lenslet array itself, and the fibre run including the slit. These will be described in turn. An overview of the KOALA instrument is given in Table 1.

\subsection{Fore-optics}

KOALA will have two sets of interchangeable fore-optics to provide two different spatial resolutions and fields of view; either 0.70" pixels and 430 sq. arcsec field-of-view, or 1.25 " pixels and 1390 sq. arcsec. field-of-view. A Zemax ray diagram of the two sets of fore-optics is shown in Figure 1, and drawings of the mechanical design are shown in Figure 2. The two fore-optics will be changed automatically, allowing the user to take advantage of periods of good seeing, or to maximise the field-of-view, depending on conditions and the science requirements.

\subsection{Lenslet array}

The KOALA IFU will consist of two telecentric hexagonally packed arrays of 1000 lenslets, both with a $250 \mu \mathrm{m}$ pitch between lenslets. The first will have hexagonal lenses which feed light to a second array of circular lenses. This double lenslet array was chosen to minimise the risk in manufacturing single-lens arrays which require specifications outside of any vendors advertised capabilities. A schematic of the optical path for a single microlens is shown in Figure 3, and photographs of prototype microlens arrays are shown in Figure 4.

The lenslet arrays will be arranged in a $40 \times 25$ configuration, giving an aspect ratio of 0.73 . The configuration of the elements has been chosen to match the expected aspect ratios of typical science targets. Figure 5 shows a histogram of the aspect ratios of all objects in the 2MASS Large Galaxy Atlas; ${ }^{11}$ the mean aspect ratio is 0.60 and the standard deviation is 0.25 . The instrument will be mounted at the Cassegrain focus which allows rotation. 

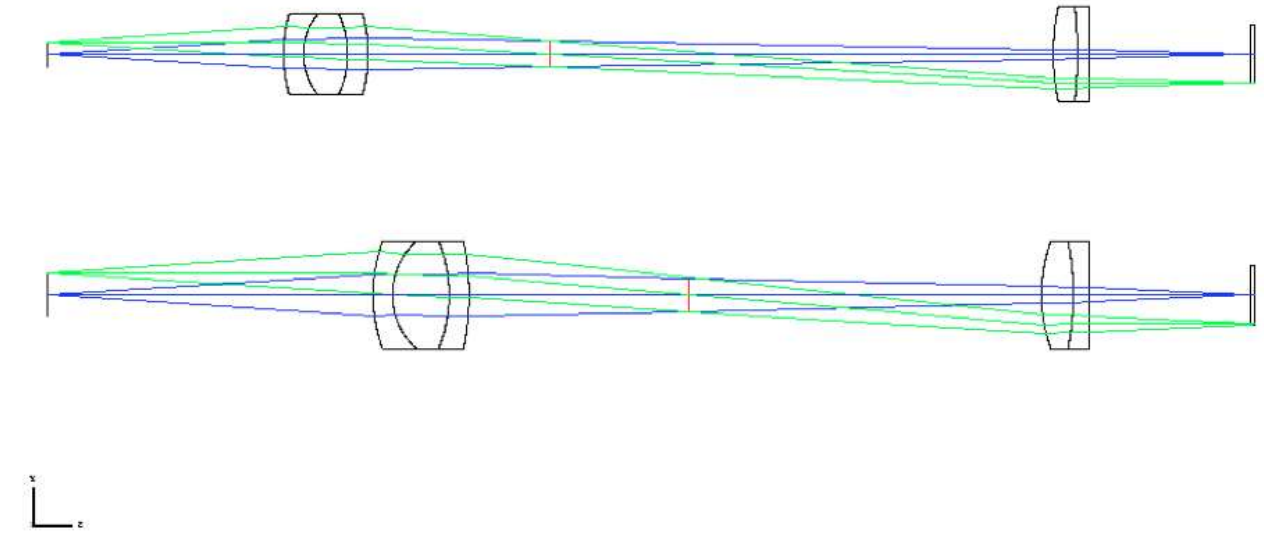

\begin{tabular}{l|l}
\hline \multicolumn{2}{c}{ 3D Layout } \\
\hline $\begin{array}{l}\text { KOALA Fore Optics } \\
8 / 12 / 2011\end{array}$ & \\
\end{tabular}

Figure 1. A Zemax diagram of both set of fore-optics, showing from left to right the telescope focal plane, lens 1, pupil stop, lens 2 , and the front surface of the lenslet array. The top image are the 0.70 scale optics and the bottom image are the 1.25 scale optics.

\subsection{Fibre run and slit}

The lenslet array will feed a $31 \mathrm{~m}$ fibre run, which will be routed down one of the telescope coudé tunnels to the AAOmega spectrograph. The fibres will be CeramOptec Optran WF 105/125/250 A, with an acrylate jacket, and provide a substantial part of the increased throughput of KOALA over SPIRAL.

The fibres will be mapped onto the slit as illustrated in Figure 6. This mapping has adjacent fibres on the IFU also adjacent on the slit, and also has adjacent blocks of 25 fibres. This reduces the chance of high contrast between adjacent fibres on the slit, minimising the fibre-to-fibre cross talk.

The blocks of 25 fibres will be housed in individual furcation tubes, all of which will be protected together in a single conduit. The 25-fibre blocks will each go to an individual slitlet on the slit. A drawing of the end slit is shown in Figure 7 . The minimum separation of the fibres along the slit is $\approx 140 \mu \mathrm{m}$. This separation equates to a cross-talk of $\approx 8 \%$ for adjacent fibres, a less than $1 \%$ for every other fibre, assuming equal illumination. This level of cross-talk can be deconvolved using the algorithms within the 2dfDR data reduction code, developed for SPIRAL observations. ${ }^{12}$

\section{EXPECTED PERFORMANCE}

The improvements in performance of KOALA over SPIRAL come from improved transmission in the fore-optics glasses, improved transmission in the fibres, better coupling of the microlenses to the fibres (i.e. better matching of the input focal ratio), and larger field-of-view. Figure 8a shows a plot of the internal transmission of the KOALA fore-optics compared to the SPIRAL fore-optics, excluding any Fresnel losses. Similarly Figure 8b shows the internal transmission of the fibres, and Figure 8c shows the combined effect of the fore-optics and fibres. Figure 8d shows the total improvement in efficiency, including a factor 1.2 improvement in the focal ratio matching of the beam injected into the fibres.

We have also estimated the absolute efficiency of KOALA including reflection losses. The results are shown in Table 2. 


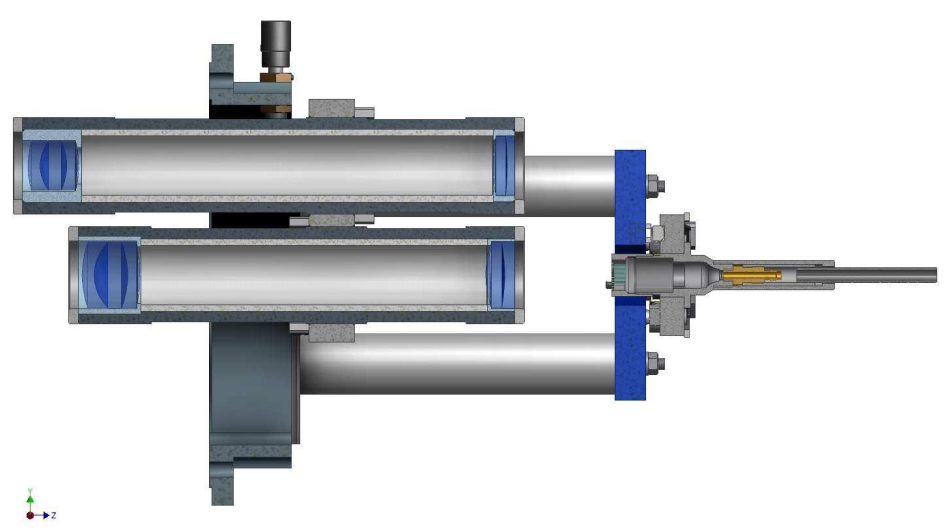

(a)

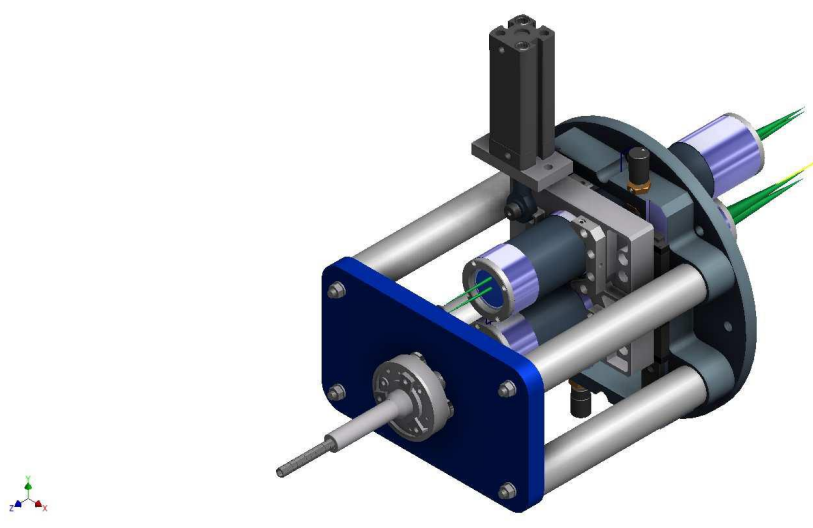

(b)

Figure 2. Drawings of the mechanical design of the fore-optics showing a profile view (a) and an outside view (b) of the two sets of fore-optics.

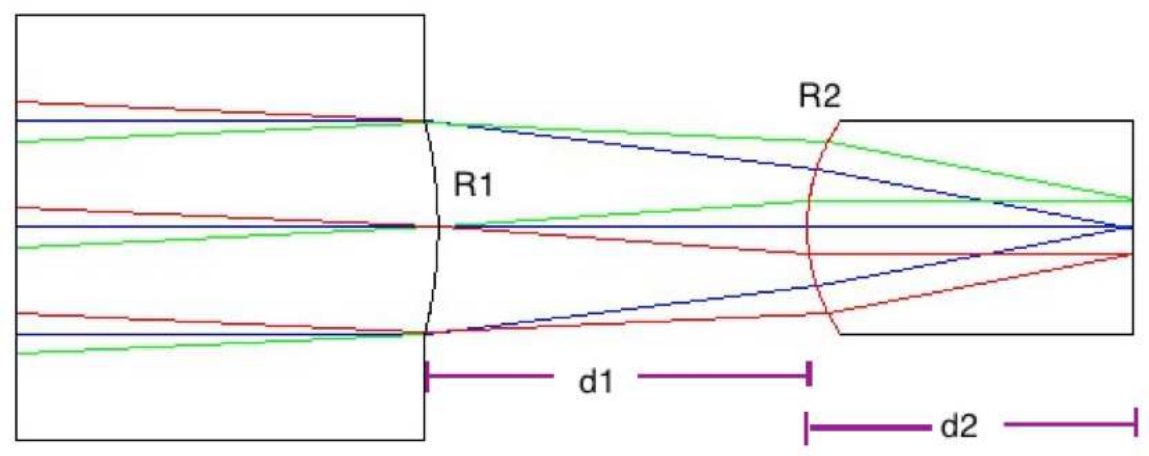

Figure 3. A schematic representation of a single element of the telecentric double lenslet array. 

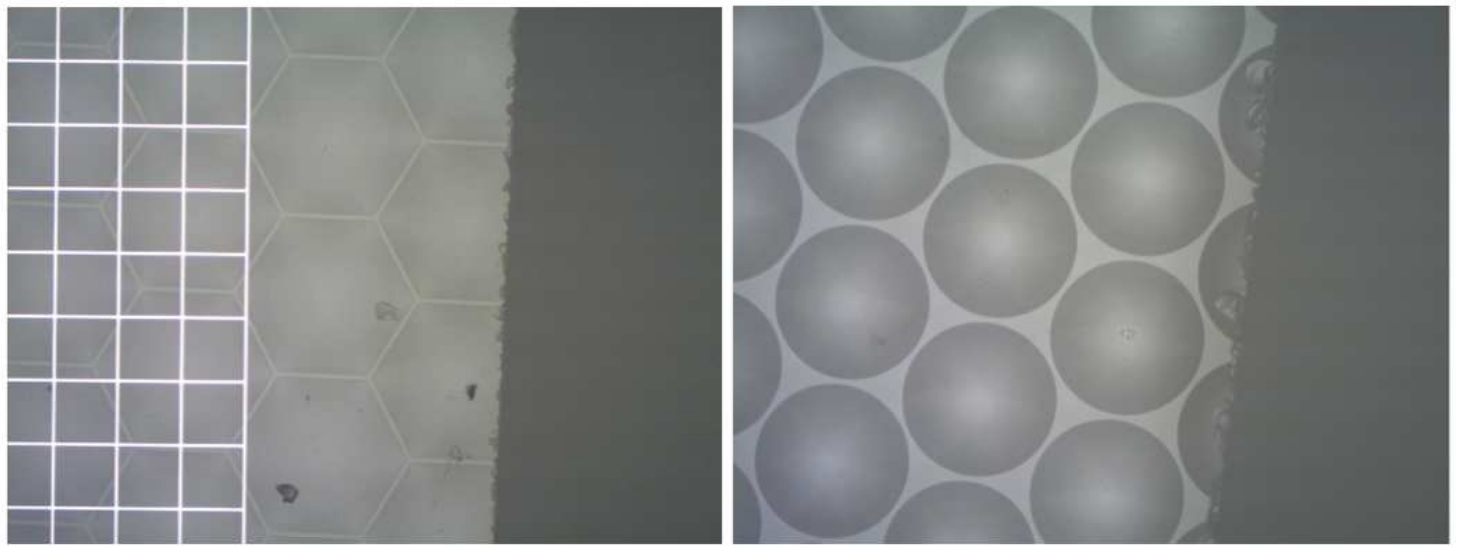

Figure 4. Photographs of prototype hexagonal and circular lenslet arrays purchased from SUSS MicroOptics. A $100 \mu \mathrm{m}$ grid is overlaid on the hexagonal array.

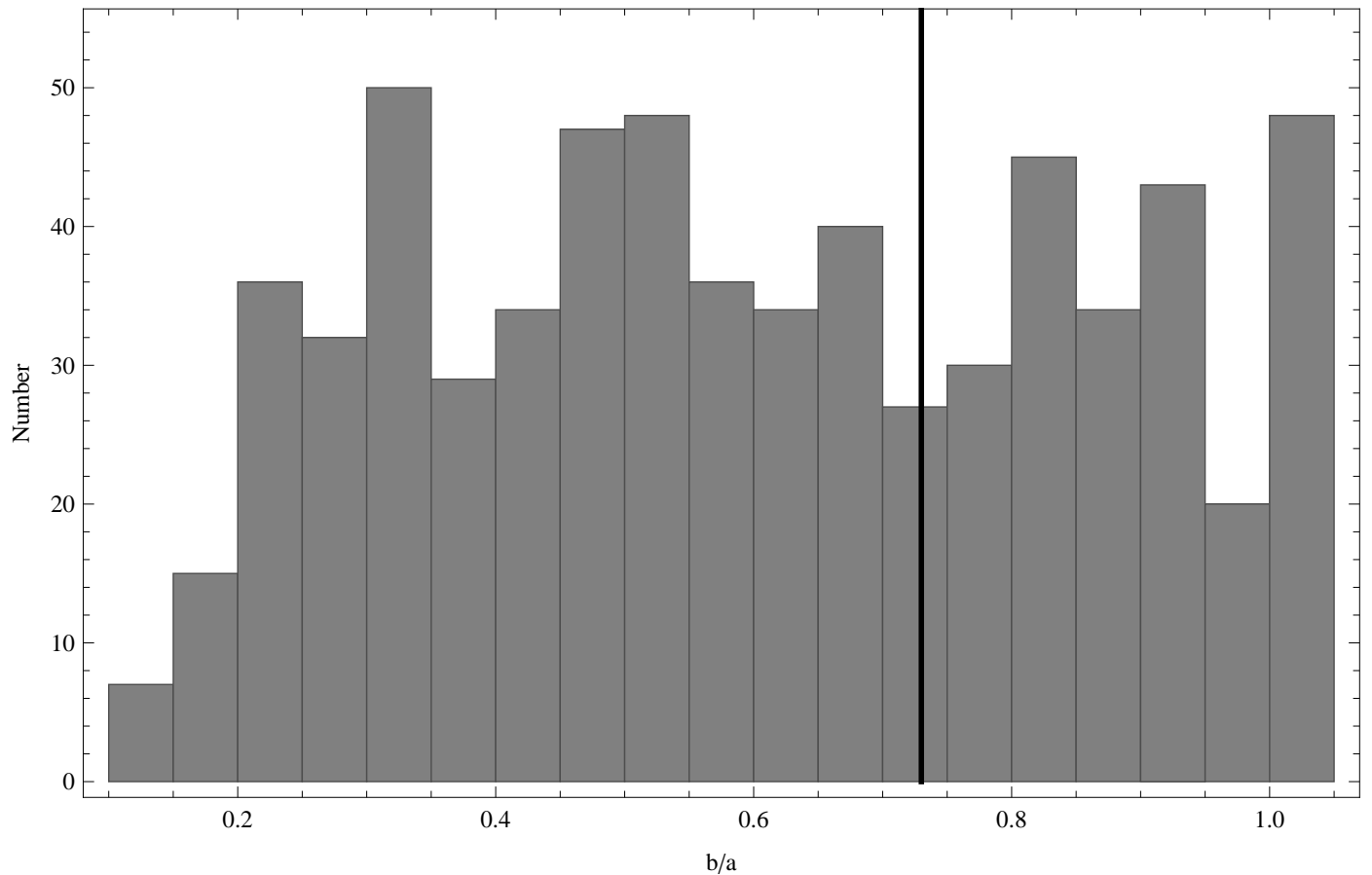

Figure 5. The ratio of minor to major axis for all objects (mostly galaxies) in the 2MASS Large Galaxy Atlas. The thick black line shows the aspect ratio of the KOALA IFU. 


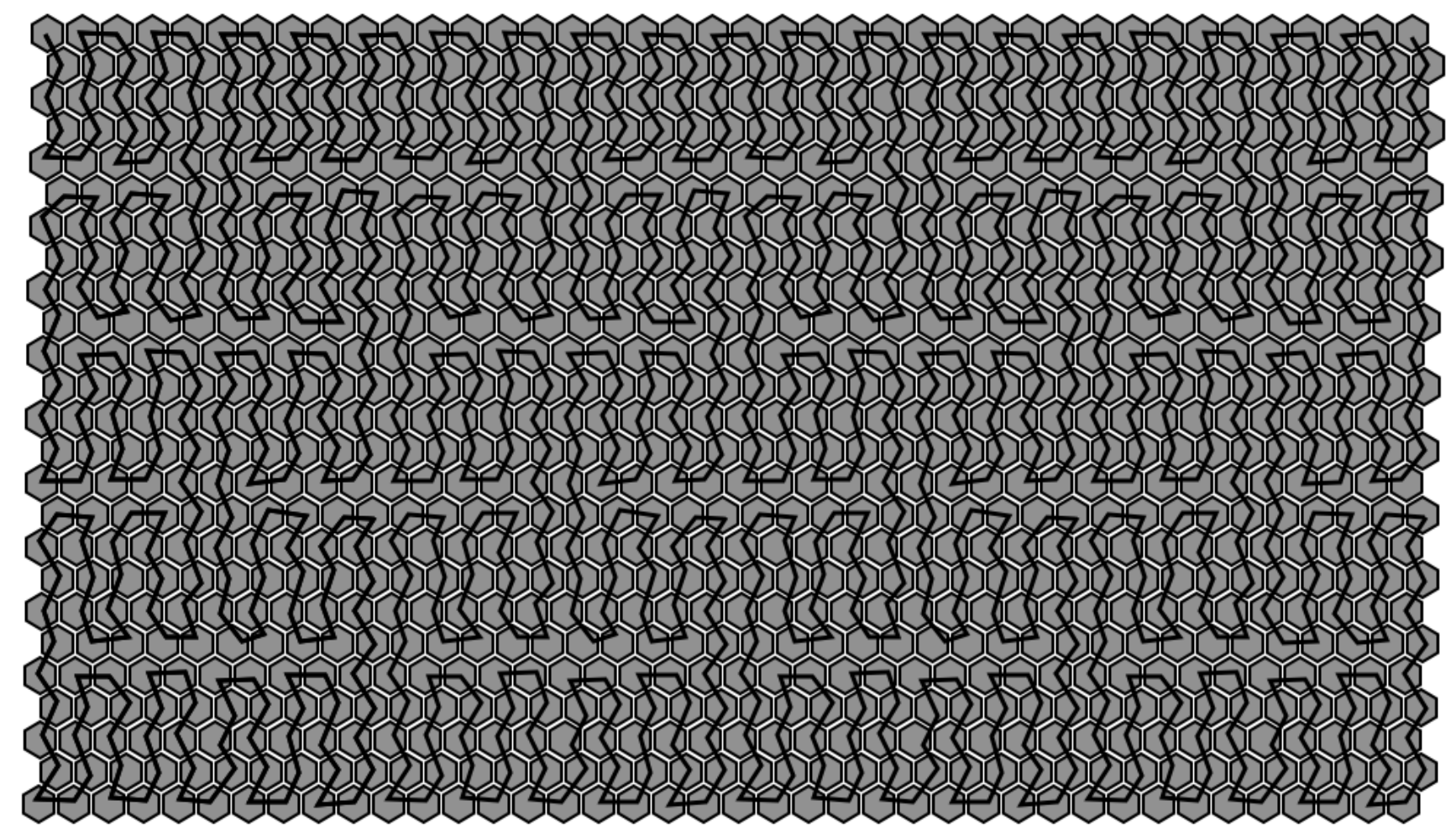

Figure 6. The mapping of fibres from the IFU to the slit follows the thick black line, starting at top left, and ending at the top right.

Table 2. The estimated total throughput of KOALA, excluding the AAOmega spectrograph.

\begin{tabular}{lll} 
Element & $3727 \AA$ & $6563 \AA$ \\
\hline Lens 1 & 0.97 & 0.97 \\
Lens 2 & 0.97 & 0.97 \\
Microlens array 1 & 0.97 & 0.97 \\
Microlens array 2 & 0.97 & 0.97 \\
Fibre & 0.82 & 0.97 \\
FRD & 0.75 & 0.75 \\
Slit & 0.99 & 0.99 \\
Mis-alignment & 0.98 & 0.98 \\
Total & 0.52 & 0.66
\end{tabular}




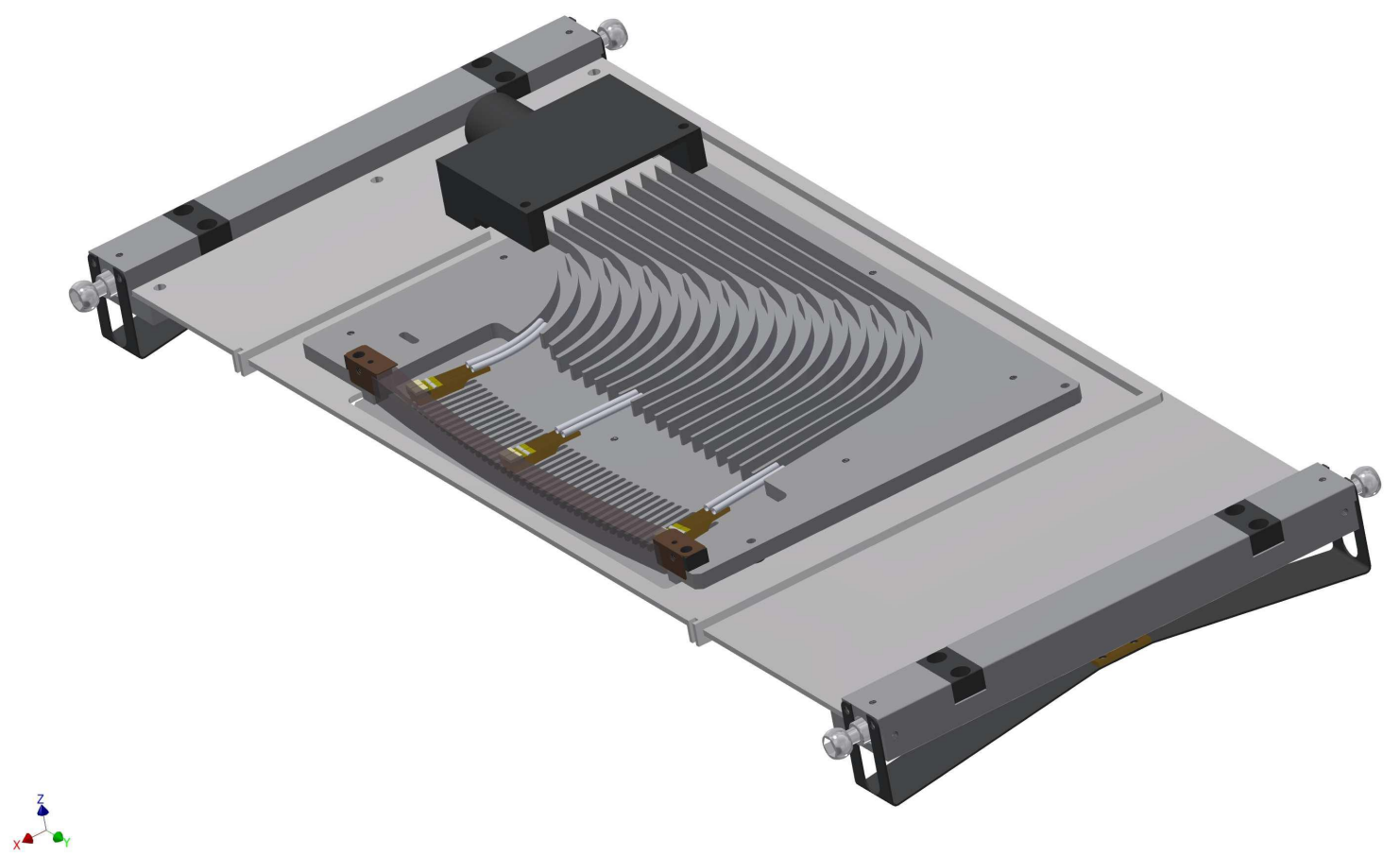

Figure 7. A drawing of the KOALA slit assembly, showing three slitlets of 25 fibres each.

\section{ACKNOWLEDGMENTS}

KOALA is funded by an ARC LIEF grant with further contributions from Macquarie University, The University of Sydney, Swinburne University of Technology, Australian National University, the University of Melbourne and the Australian Astronomical Observatory.

\section{REFERENCES}

[1] Lee, D. and Taylor, K., "Fiber developments at the anglo-australian observatory for spiral and australis," Proc. SPIE 4008, 268 (2000).

[2] Parry, I. R., Kenworthy, M., and Taylor, K., "SPIRAL Phase A: a prototype integral field spectrograph for the AAT," Proc. SPIE 2871, 1325-1331 (Mar. 1997).

[3] Kenworthy, M. A., Parry, I. R., and Taylor, K., "SPIRAL Phase A: A Prototype Integral Field Spectrograph for the Anglo-AustralianTelescope," PASP 113, 215-226 (Feb. 2001).

[4] Saunders, W., Bridges, T., Gillingham, P., Haynes, R., Smith, G. A., Whittard, J. D., Churilov, V., Lankshear, A., Croom, S., Jones, D., and Boshuizen, C., "AAOmega: a scientific and optical overview," Proc. SPIE 5492, 389-400 (Sept. 2004).

[5] Smith, G. A., Saunders, W., Bridges, T., Churilov, V., Lankshear, A., Dawson, J., Correll, D., Waller, L., Haynes, R., and Frost, G., "AAOmega: a multipurpose fiber-fed spectrograph for the AAT," Prc. SPIE 5492, 410-420 (Sept. 2004).

[6] Sharp, R., Saunders, W., Smith, G., Churilov, V., Correll, D., Dawson, J., Farrel, T., Frost, G., Haynes, R., Heald, R., Lankshear, A., Mayfield, D., Waller, L., and Whittard, D., "Performance of AAOmega: the AAT multi-purpose fiber-fed spectrograph," Proc. SPIE 6269, 14 (July 2006).

[7] Green, A. W., Glazebrook, K., McGregor, P. J., Abraham, R. G., Poole, G. B., Damjanov, I., McCarthy, P. J., Colless, M., and Sharp, R. G., "High star formation rates as the origin of turbulence in early and modern disk galaxies," Nature 467, 684-686 (Oct. 2010). 


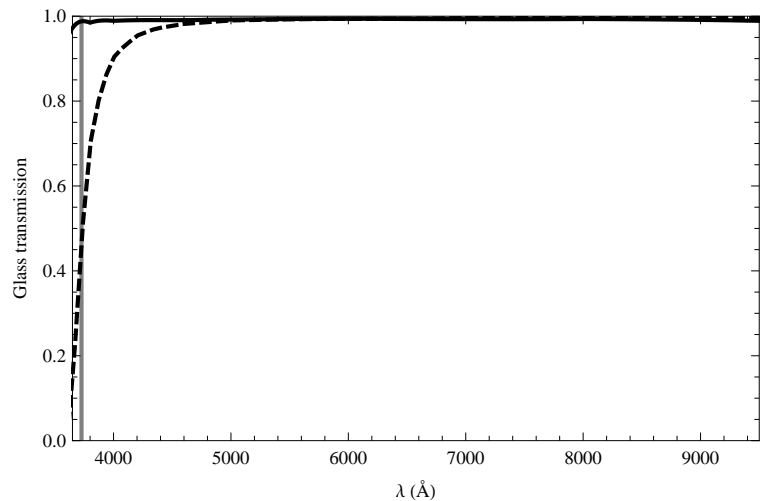

(a) Fore-optics

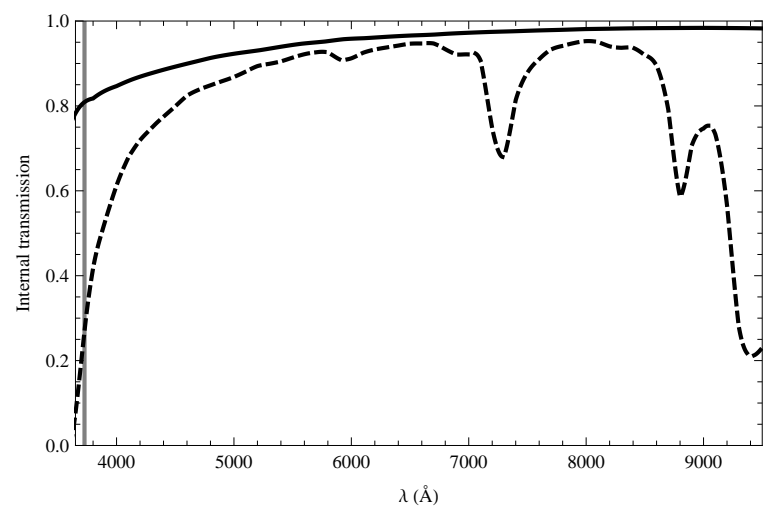

(c) Total internal transmission

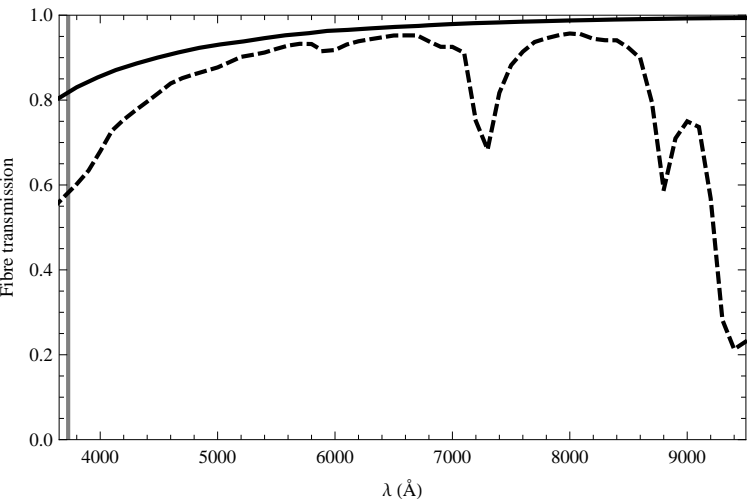

(b) Fibre

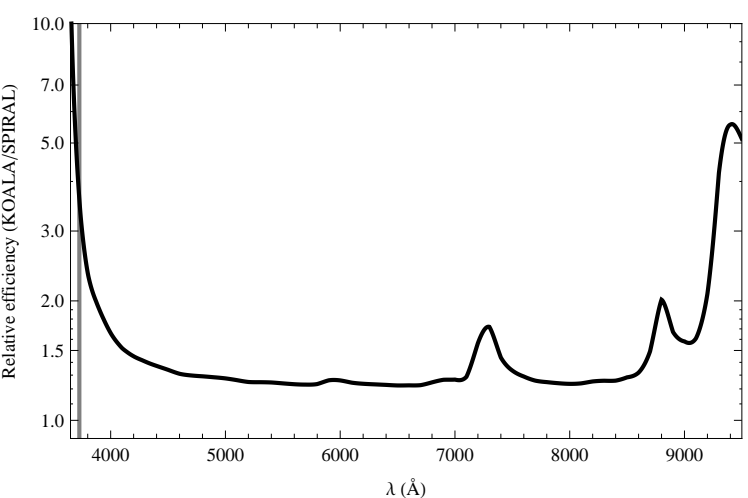

(d) Relative efficiency (KOALA/SPIRAL)

Figure 8. The internal losses of the fore-optics and fibres for KOALA (continuous line) and SPIRAL (dashed line) as a function of wavelength. The vertical grey line marks the position of [OII] $3737 \AA$. The bottom plots show the overall improvement in internal transmission.

[8] Sharp, R. G. and Bland-Hawthorn, J., "Three-Dimensional Integral Field Observations of 10 Galactic Winds. I. Extended Phase of Mass/Energy Injection Before the Wind Blows," ApJ 711, 818-852 (Mar. 2010).

[9] Bacon, R., Copin, Y., Monnet, G., Miller, B. W., Allington-Smith, J. R., Bureau, M., Carollo, C. M., Davies, R. L., Emsellem, E., Kuntschner, H., Peletier, R. F., Verolme, E. K., and de Zeeuw, P. T., "The SAURON project - I. The panoramic integral-field spectrograph," MNRAS 326, 23-35 (Sept. 2001).

[10] Cappellari, M., Emsellem, E., Krajnović, D., McDermid, R. M., Scott, N., Verdoes Kleijn, G. A., Young, L. M., Alatalo, K., Bacon, R., Blitz, L., Bois, M., Bournaud, F., Bureau, M., Davies, R. L., Davis, T. A., de Zeeuw, P. T., Duc, P.-A., Khochfar, S., Kuntschner, H., Lablanche, P.-Y., Morganti, R., Naab, T., Oosterloo, T., Sarzi, M., Serra, P., and Weijmans, A.-M., "The ATLAS ${ }^{3 D}$ project - I. A volume-limited sample of 260 nearby early-type galaxies: science goals and selection criteria," MNRAS 413, 813-836 (May 2011).

[11] Jarrett, T. H., Chester, T., Cutri, R., Schneider, S., and Huchra, J. P., "The 2mass large galaxy atlas," AJ 125, 525 (2003).

[12] Sharp, R. and Birchall, M. N., "Optimal Extraction of Fibre Optic Spectroscopy," PASA 27, 91-103 (Mar. 2010). 